\title{
La eficacia de la reexperimentación emocional: el papel del optimismo y la alexitimia
}

\author{
Tasmania del Pino ${ }^{1}$, Wenceslao Peñate ${ }^{1}$, Ascensión Fumero ${ }^{1}$, Juan Manuel \\ Bethencourt ${ }^{1}$ y Sonia Zambrano ${ }^{2}$ \\ ${ }^{1}$ Universidad de La Laguna (España); ${ }^{2}$ Universidad Católica de Colombia (Colombia)
}

Los efectos beneficiosos sobre la salud de la reexperimentación emocional mediante la utilización de la escritura expresiva de vivencias traumáticas están bien establecidos. La efectividad de la utilización de la reexperimentación parece verse afectada por ciertas condiciones. Entre las variables personales que han mostrado tener influencia sobre la recuperación de tales vivencias se ha destacado el optimismo y la alexitimia. El objetivo del presente trabajo fue evaluar los efectos de una intervención basada en la reexperimentación emocional sobre la salud mental. Se aplicó un diseño experimental intergrupo pre y post-tratamiento. La muestra estuvo compuesta por un total de 60 participantes, de los que 40 llevaron a cabo un procedimiento de reexperimentación emocional mediante tres sesiones de escritura expresiva. El resto formaron el grupo control y escribieron sobre situaciones triviales. Todos fueron evaluados antes y después de la reexperimentación emocional en afecto positivo y negativo, ansiedad, depresión, pensamientos intrusivos y evitación cognitiva. Además, se controló el nivel de optimismo y la alexitimia. Los resultados mostraron la eficacia de la escritura expresiva sobre el afecto positivo y negativo y el nivel de depresión. Entre las covariables, únicamente el optimismo produjo un efecto significativo sobre las variables más emocionales. Se discuten las condiciones contextuales y personales que pueden favorecer el uso clínico de la reexperimentación emocional.

Palabras clave: Reexperimentación emocional, escritura expresiva, salud mental, alexitimia, optimismo.

The efficacy of expressive writing intervention: the role of optimism and alexithymia. The beneficial effects on the health of the re-experimentation of traumatic experiences are well-established. The effectiveness of using reexperimentation seems to be affected by certain conditions. Among personal variables that have been shown to influence the recovery of such experiences it has highlighted the optimism and alexithymia. The aim of this study was to evaluate the effects on mental health of an intervention based on re-experimentation. The sample consisted in a total of 60 participants, of which 40 carried out a process of emotional re-experimentation through three sessions of expressive writing. The rest formed the control group. All participants were evaluated before and after the emotional disclosure in positive and negative affect, anxiety, depression, intrusive thoughts and cognitive avoidance. Furthermore, the level of optimism and alexithymia were controlled. The results showed the effectiveness of expressive writing on the positive and negative affect and on depression. Among the covariables, only optimism affected significantly to effect emotional variables. The contextual and personal conditions that can be ameliorated the clinical use of emotional disclosure were discussed.

Keywords: Emotional disclosure, expressive writing, mental health, alexithymia, optimism.

Correspondencia: Wenceslao Peñate. Facultad de Ciencias de la Salud - Sección Psicología. Universidad de La Laguna. Campus de Guajara. C.P.: 38204. Tenerife (España). E-mail: wpenate@ull.edu.es 
La escritura expresiva describe un tipo de narración sobre acontecimientos y sentimientos derivados de situaciones traumáticas, estresantes o emocionales. El paradigma de investigación centrado en la escritura expresiva ha evidenciado los efectos beneficiosos que la recuperación y reexperimentación emocional (RE) de traumas vividos produce sobre la salud física y el ajuste psicológico (Baikie, Geerlings, y Wilhelm, 2012).

En términos de eficacia general, en diferentes comunidades y muestras de enfermos, se han encontrado evidencias de beneficios significativos en la salud emocional, la salud física percibida, cambios positivos en el sistema inmunológico y en el funcionamiento psicológico general después de la comunicación emocional (Frattaroli, 2006; Lumley et al., 2014). Sin embargo, los resultados son menos consistentes para los participantes con dificultades psicológicas (Baikie et al., 2012; Travagin, Margola, y Revenson, 2015). Mientras algunos estudios han mostrado apoyo para la escritura expresiva en estudiantes con una historia de trauma (Ironson et al., 2013), los beneficios se han mostrado limitados en muestras de participantes con imagen negativa del propio cuerpo (Lafont y Oberle, 2014; O'Connor et al., 2011), cuidadores de pacientes con enfermedades crónicas (Butcher et al. 2016), adultos que han sufrido abuso infantil, que presentan síntomas de depresión y estrés postraumático o que habían experimentado la pérdida de un familiar (Baikie et al., 2012; Meston, Lorenz, y Stephenson, 2013; Unterhitzenberger y Rosner, 2014). Llegando incluso a mostrarse efectos paradójicos, en el sentido de un mayor agravamiento de los síntomas en el grupo de RE unido a peor nivel de satisfacción vital (Meads y Nouwen, 2005).

Se ha informado de mayor eficacia de la RE en hombres, en universitarios y en los casos en que se evocaban traumas actuales, observándose un posible efecto iatrogénico inicial que, en general, se va disipando poco a poco (Smyth, 1998). Uno de los estudios más amplios de meta-análisis demostró que los participantes que tenían un mayor estrés, peor salud física y menor optimismo antes de realizar la escritura expresiva experimentaron mayores beneficios de la RE, asociados a tamaños del efecto de mayor importancia (Frattaroli, 2006). Una vía de explicación de las razones por las que la RE funciona se encuentra en que activa un proceso de aprendizaje autorregulado, definido como un proceso activo por el que las personas monitorizan, regulan y controlan sus pensamientos y sus motivaciones (Núñez, Amieiro, Álvarez, García, y Dobarro, 2015). Al analizar las variables que pueden modular estos resultados se observó que los participantes que obtuvieron mayores beneficios de la divulgación emocional escrita fueron aquellos que se enfrentaban a malestar debido a problemas emocionales tales como experiencias traumáticas o problemas de salud recurrentes, en las situaciones en que la apertura emocional se realizaba en privado, con problemas recientes, con instrucciones específicas sobre qué reexperimentar, con al menos 3 sesiones de una duración mínima de 15 minutos y un seguimiento mínimo de un mes. También es 
posible entender que el modo en que las personas acceden a sus recuerdos, la facilidad para redactar esos eventos y el modo de afrontarlos positivamente pueden tener que ver con su capacidad intelectual, especialmente aquella que se deriva de procesos de sabiduría (i.e., modelo WICS, Sternberg, 2015).

Por otra parte, en la revisión de Merz, Fox, y Malcorn (2014) basada en ensayos clínicos con pacientes oncológicos se observaron pocos resultados significativos en la calidad del sueño, el dolor y algunos síntomas psicológicos. Los tamaños del efecto alcanzaron niveles que fueron desde bajos hasta moderados. En este caso, las limitaciones sociales y los niveles elevados de neuroticismo se identificaron como moduladores que disminuían el efecto beneficioso de la RE. Aunque los estudios revisados presentaban varias limitaciones como la variabilidad en el cáncer (tipo y estadio) o la heterogeneidad del protocolo de RE utilizado. En suma, la RE puede considerarse una intervención eficaz para promover la regulación emocional y el bienestar de las poblaciones vulnerables, especialmente teniendo en cuenta la relación coste-beneficio. Sin embargo, la gran variabilidad en el tamaño del efecto en función de la medida de resultado o la población estudiada sugiere la necesidad de investigar los moduladores potenciales y definir las condiciones en las que debe tener lugar la RE.

Puesto que no todo el mundo se beneficia por igual del proceso de escritura expresiva, algunos participantes incluso parecen empeorar, más allá del efecto iatrogénico pasajero que supone recordar una vivencia traumática, el interés de la investigación se ha dirigido hacia la identificación de determinadas variables moduladoras. En este sentido, se han analizado determinadas variables contextuales, como el tipo de instrucción, la duración, número de sesiones, o el tipo de trauma a reexperimentar (Peñate, del Pino-Sedeño, y Bethencourt, 2010) y variables de carácter personal, como el optimismo y la alexitimia (Baikie, 2008; Frattaroli, 2006).

La investigación sobre afrontamiento de situaciones estresantes indica que pensar y planear utilizando las emociones positivas y el optimismo resulta saludable ante las fuentes crónicas e inminentes de estrés (Taber, Klein, Ferrer, Kent, y Harris, 2016). Aunque los efectos de la RE se han mostrado más fuertes para los pesimistas probablemente debido a que los optimistas suelen afrontar más adecuadamente los problemas (Clyman y Pachankis, 2014). Mackenzie, Wiprzycka, Hasher, y Goldstein (2008) encontraron apoyo a la hipótesis de que la RE debe ser positiva, optimista y orientada hacia el futuro para mejorar la salud de los cuidadores de personas dependientes. Además, los participantes que escribieron sobre sus circunstancias difíciles se beneficiaron más en términos de salud y elevaron sus puntuaciones en optimismo.

Por otro lado, las personas alexitímicas tienen dificultades para identificar y etiquetar sus emociones (Peasley-Miklus, Panayiotou, y Vrana, 2016). Estudios previos mostraron que las personas con dificultades para describir los sentimientos obtenían 
mayores beneficios de la escritura expresiva en términos de visitas al médico por enfermedad, síntomas de depresión y trastornos del sueño (Krpan et al., 2013). Kállay (2015) destacó que una verbalización pobre parecía estar asociada con problemas en el estado de ánimo y alta percepción de síntomas físicos. Cuando se han logrado superar estas dificultades, las personas con altos niveles de alexitimia se beneficiaron más de un procedimiento de RE (Baikie, 2008). Por lo que parece que la RE puede proporcionar una oportunidad para que los individuos alexitímicos puedan procesar el material emocional sin que se precise la interacción interpersonal de las terapias habladas (Ashley, O’Connor, y Jones, 2011; Niles, Haltom, Mulvenna, Lieberman, y Stanton, 2014). Estos resultados sugieren que la escritura expresiva tiene ventajas particulares para los individuos que puntúan alto en alexitimia y puede proporcionarles la oportunidad de procesar el material traumático de una manera segura, es decir, les permite controlar cuánto se dan a conocer. Este hecho favorece determinar de una manera más eficaz su propia dosis (Pennebaker, 2002). Estos resultados apuntan a la conveniencia de incluir medidas de personalidad en los estudios sobre escritura expresiva, para que se pueda entender mejor cuándo y para quién funciona de una manera más adecuada la escritura expresiva (Ashley et al., 2011).

En el presente estudio se ha planteado como objetivo detectar si los efectos de la RE sobre la salud psicológica podrían estar modulados por los niveles de alexitimia y de optimismo. Específicamente, se pretende contrastar los resultados obtenidos por un grupo experimental que lleva a cabo una RE con los de un grupo control en las variables de afecto positivo y negativo, ansiedad, depresión y pensamientos intrusivos y evitativos, manteniendo constantes las variables personales de alexitimia y optimismo para observar su papel modulador sobre la RE. Además, se intentará comprobar si las emociones se ven afectadas en las distintas sesiones experimentales.

\section{MÉTODO}

\section{Participantes}

La muestra final que completó todas las sesiones de reexperimentación emocional y el seguimiento estuvo compuesta por 60 estudiantes voluntarios de la Universidad de La Laguna.

Tabla 1. Estadísticos descriptivos de la muestra general y de la condición experimental (escribir sobre situaciones traumáticas, sesión a sesión) y control

\begin{tabular}{lccc}
\hline & Muestra completa & Grupo experimental & Grupo control \\
\hline $\mathrm{N}^{\circ}$ de participantes & 60 & 40 & 20 \\
\hline Edad media & 21.7 años & 21.8 & 21.5 \\
\hline Rango de edad & $19-30$ años & $20-29$ años & $19-30$ años \\
\hline \multirow{2}{*}{ Género } & $10 \%$ hombres & $5 \%$ hombres & $10 \%$ hombres \\
& $90 \%$ mujeres & $95 \%$ mujeres & $90 \%$ mujeres \\
\hline
\end{tabular}


Participaron 54 mujeres y 6 hombres con una media de edad de 21.7 años. El rango de edad estuvo entre 19 a 30 años (73\% tenía entre 20 y 22 años). En la tabla 1 se muestra un resumen de los datos más relevantes.

\section{Instrumentos}

Escala de afecto positivo y negativo (PANAS; Watson, Clark, y Tellegen, 1988) contiene 20 descriptores de estados de ánimo (diez positivos y diez negativos). Esta escala muestra una estructura bifactorial compuesta por dos subescalas independientes, afecto positivo y afecto negativo. Se han mostrado índices de fiabilidad que alcanzan 90 para el afecto positivo y .87 para el negativo (Nolla, Queral, y Miró, 2014). En este estudio se ha utilizado para monitorizar el estado de ánimo sesión a sesión.

Escala sobre impacto de los acontecimientos (IES; Horowitz, Wilner, y Álvarez, 1979) formada por 15 ítems dicotómicos relacionados con la vivencia de situaciones traumáticas: pensamientos intrusivos asociados con el trauma y conductas evitativas relacionadas con el hecho vivido. La versión española de la escala alcanzó buenos ajustes psicométricos, $\alpha=.94, .93$ y .85; coeficientes test-retest=.35, .36, .28 para las puntuaciones totales, los pensamientos intrusivos y las conductas evitativas, respectivamente (Baguena et al., 2001).

Escala de Ansiedad y Depresión Hospitalaria (HADS; Zigmond y Snaith, 1983) consta de 14 ítems (siete de ansiedad y siete de depresión) distribuidos en dos subescalas. Se centra en los aspectos emocionales y cognitivos de estos dos trastornos. La versión española de la escala ha mostrado unos ajustes psicométricos adecuados, $\alpha=.74, .59$, y .76 para ansiedad, depresión y escala total, respectivamente (Gil, Oberst, Del Valle, y Chamarro, 2015).

Test de Orientación Vital revisado (LOT-R; Scheier, Carver, y Bridge, 1994) consta de 8 ítems más 4 de relleno y evalúa las expectativas generalizadas positivas y negativas sobre los resultados en la vida y representa una medida del nivel de optimismo. La adaptación española obtiene una estimación de la consistencia interna de 0.7 (Vera-Villarroel y Celis-Atenas, 2014).

Escala de Alexitimia de Toronto (TAS-26; Taylor, Bagby, y Parker, 1997) evalúa el nivel de alexitimia entendida como la dificultad para describir sentimientos y emociones por medio de 26 ítems. La escala resultó consistente, $\alpha=.82$, y estable a los 6 meses, $r=.71$ (Arancibia y Behar, 2015).

\section{Diseño}

Se realizó un diseño experimental intrasujeto con medidas antes y después de la intervención, con una variable intersujetos que consistía en la narración escrita de un trauma o suceso estresante doloroso o la narración de situaciones triviales. La 
intervención consistió en tres sesiones de escritura de 30 minutos de duración en días consecutivos. Los participantes fueron asignados aleatoriamente a la condición experimental y debían escribir sobre situaciones estresantes o traumáticas o a la condición de grupo control escribiendo sobre cuestiones triviales. Las variables dependientes utilizadas fueron afecto positivo y negativo, ansiedad, depresión, pensamientos intrusivos y de evitación. Además, se controló el efecto de dos covariables, alexitimia y optimismo. La evaluación del afecto positivo y negativo se realizó después de cada sesión experimental.

\section{Procedimiento}

Los participantes fueron citados individualmente. Se les informaba que tendrían que escribir sobre una situación personal. Se les comunicó que los cuestionarios y materiales serían tratados confidencialmente. Una vez firmado el consentimiento informado, se les suministró el dossier de pruebas y las instrucciones adecuadas a la condición experimental a la que se les asignaba. El grupo experimental recibió las siguientes instrucciones: "Los seres humanos estamos expuesto a una serie de hechos que no podemos controlar y que nos suponen un cierto daño psicológico (accidentes de tráfico, desastres naturales, muertes de seres querido, observar la muerte o el suicidio de alguien, ser asaltado, sufrir abusos sexuales, ser violada, etc.). Ahora trata de recordar un hecho que te haya ocurrido o que hubieras presenciado y que te haya resultado altamente molesto, muy estresante o traumático. Preferiblemente céntrate en el que te haya parecido más traumático. Concéntrate en todos los detalles (sonidos, olores, personas, lugares, colores, etc.), cierra los ojos si ello te ayuda a concentrarte. Haz pasar esa historia por tu mente y, a continuación, descríbela durante 30 minutos, escribiendo todos los detalles que recuerdes, acompañado de tus sensaciones y tus emociones. Escribe desde lo que sucedió antes del hecho hasta después de pasado el mismo. No te preocupes por la ortografía, la sintaxis o la gramática, solo es interesante que describas tus sentimientos y emociones más profundas asociada a esos hechos".

Por otro lado, las instrucciones para el grupo control fueron las siguientes: "Durante 30 minutos, trata de recordar cronológicamente todo lo que has hecho hoy, desde que te levantaste hasta que llegaste aquí. Concéntrate y haz pasar el desarrollo de este día por tu mente y, a continuación, escríbelo, describiendo todos los detalles que recuerdes, acompañados de tus sensaciones y tus emociones. No te preocupes por la ortografía, la sintaxis o la gramática".

Inmediatamente después de cada una de las tres sesiones experimentales cumplimentaban la escala PANAS. 


\section{Análisis de datos}

Se aplicó un análisis de varianza de medidas repetidas (MANOVA) para conocer los efectos de la RE antes y después de la intervención. Asimismo, se contrastaron estos efectos entre los grupos experimental y control. Además, se realizó un análisis mutivariado de la varianza (MANCOVA) para identificar el papel de la alexitimia y el optimismo como covariables (siempre que un participante tuvo un dato perdido, fue eliminado en esa variable para los análisis, por lo que los grados de libertad pueden variar).

\section{RESULTADOS}

En la tabla 2 se presentan los datos descriptivos en las variables dependientes utilizadas.

Tabla 2. Medias y desviaciones típicas del grupo experimental $(N=40)$ y control $(N=20)$ en las variables dependientes antes y después de la intervención

\begin{tabular}{llrrrr}
\hline \multirow{2}{*}{ Variables } & \multicolumn{2}{c}{ Grupo } & \multicolumn{2}{c}{ Pretest } & \multicolumn{2}{c}{ Sesión 3/ Post-test } \\
\cline { 2 - 5 } PA & & $M$ & $D T$ & $M$ & $D T$ \\
\hline \multirow{2}{*}{ EA } & Cxperimental & 12.03 & 6.14 & 17.83 & 6.00 \\
\cline { 2 - 6 } & Experimental & 8.23 & 6.30 & 4.43 & 4.71 \\
\cline { 2 - 6 } Ansiedad & Control & 2.50 & 2.64 & 2.50 & 2.50 \\
\hline \multirow{2}{*}{ Depresión } & Experimental & 14.88 & 4.55 & 8.79 & 3.31 \\
\cline { 2 - 6 } & Control & 15.42 & 3.62 & 9.29 & 3.65 \\
\hline \multirow{2}{*}{ Pensamientos intrusivos } & Experimental & 8.18 & 3.37 & 5.43 & 3.03 \\
\cline { 2 - 6 } & Control & 7.68 & 2.85 & 7.24 & 3.42 \\
\hline \multirow{2}{*}{ Evitación cognitiva } & Experimental & 8.40 & 6.64 & 8.39 & 6.16 \\
\cline { 2 - 6 } & Control & 8.45 & 4.59 & 6.41 & 5.90 \\
\hline \multirow{2}{*}{ Eota: $M=$ Experimental } & 10.20 & 4.62 & 8.68 & 5.09 \\
\cline { 2 - 6 } & Control & 10.45 & 5.20 & 6.41 & 5.90 \\
\hline
\end{tabular}

Nota: $M=$ media; $D T=$ desviación típica; $\mathrm{PA}=$ afecto positivo; $\mathrm{NA}=$ afecto negativo.

Figura 1. Afecto positivo evaluado sesión a sesión en los grupos experimental y control

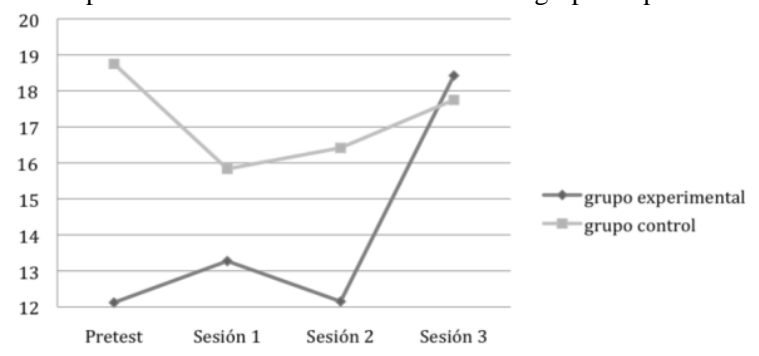

El contraste de los datos previos a la RE en relación con la igualdad entre los grupos control y experimental no mostró diferencias significativas en ninguna de las variables evaluadas. 
Con respecto a los cambios antes-después, el MANOVA sobre los dos factores de la escala PANAS indicó que en afecto positivo se obtuvo un efecto significativo de la interacción entre la condición experimental y el momento de la evaluación, $F(1,41)=6.17, p \leq .02\left(\eta^{2}=.13\right)$. La figura 1 muestra dicho efecto. Así, se observa que en el grupo experimental aumentó el afecto positivo, partiendo de niveles más bajos, mientras que las puntuaciones disminuyeron ligeramente en el grupo control.

En afecto negativo, también hubo un efecto significativo de la interacción entre la condición experimental y el momento de la evaluación, $F(1,42)=11.97, p \leq .001$ $\left(\eta^{2}=.22\right)$. Como puede observarse en la figura 2, el grupo que llevó a cabo la RE disminuyó sus puntuaciones en afecto negativo mientras que el grupo control parece mantenerlas.

Figura 2. Afecto negativo evaluado sesión a sesión en los grupos experimental y control

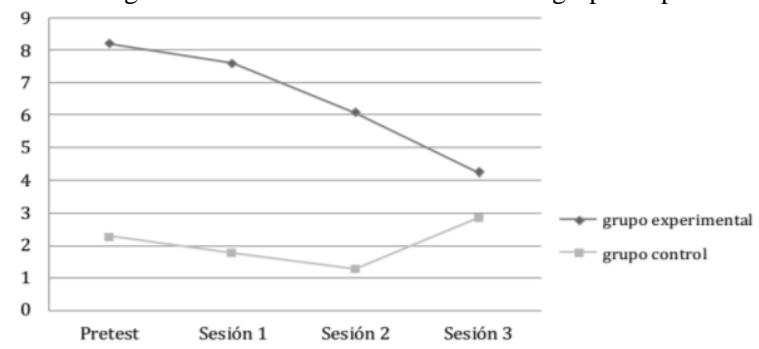

La comparación de la cuantía media de las puntuaciones para ambos grupos mostró la activación de la respuesta emocional a lo largo de las sesiones experimentales producida como consecuencia de la RE. Como puede observarse en las figuras 1 y 2, la cuantía media fue inferior en las emociones positivas y superior en las negativas en el caso del grupo experimental comparado con el grupo control.

Figura 3. Puntuaciones en depresión en las fases de pretest y post-test en los grupos experimental y control

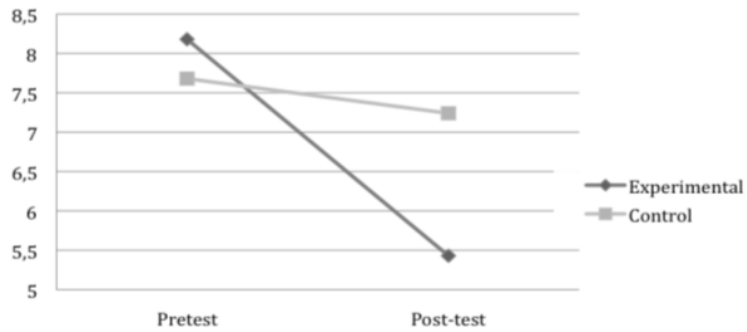

En el caso del nivel de depresión, se obtuvo una interacción significativa, $F(1,50)=9.51, p \leq .003\left(\eta^{2}=.160\right)$, que pone de manifiesto que el grupo que participó en la RE disminuyó su nivel de depresión. En la figura 3 se puede observar dicho efecto. Sin 
embargo, no se observaron efectos significativos de la RE sobre el nivel de ansiedad ni sobre los pensamientos intrusivos y evitativos.

Mediante el análisis de MANCOVA se puede observar un efecto significativo del optimismo como covariable en el caso de la ansiedad, $F(1,48)=5.14, p \leq 0.03\left(\eta^{2}=.1\right)$, observándose una relación entre disminución de la ansiedad y el optimismo. En el caso de la depresión, el optimismo alcanzó una puntuación marginalmente significativa $(p \leq .08)$. Sin embargo, la alexitimia no arrojó resultados significativos.

\section{DISCUSIÓN Y CONCLUSIONES}

Este estudio examinó la relación entre el paradigma de reexperimentación emocional, llevado a cabo a través de la escritura de situaciones traumáticas, altamente molestas o estresantes y la salud psicológica. Asimismo, se ha analizado el papel que la alexitimia y el optimismo tienen sobre los efectos beneficiosos del procedimiento de RE. Después de realizar la intervención basada en la escritura expresiva, se observaron efectos significativos de la RE sobre el afecto positivo, el afecto negativo y el nivel de depresión. Sin embargo, la ansiedad y los pensamientos intrusivos y evitativos no parecen modificarse después de la realización de la RE. El optimismo aparece implicado principalmente con las variables más emocionales (ansiedad y, en menor medida, depresión), mientras que la alexitimia no presenta un papel tan significativo.

Desde el punto de vista de la validez interna, se pudo constatar que la RE logró alterar tanto las emociones positivas como las negativas. Específicamente, se ha observado que la RE posee un efecto sobre el estado de ánimo más situacional, donde se llegan a alcanzar los niveles más adaptativos representados por un menor afecto negativo y mayor afecto positivo; datos que se encuentran en la línea de los autores que observaron que la RE se asoció a mejoras en el estado de ánimo tanto en estudiantes saludables como en muestras clínicas (Baikie et al., 2012; Blasio et al., 2015; Krpan et al., 2013). Estos efectos contextuales se ven corroborados por la disminución de los niveles de depresión en el grupo que llevó a cabo la RE.

Sin embargo, los datos obtenidos sobre el nivel de ansiedad muestran la eficiencia del paso del tiempo y no parecen afectados por la utilización de la escritura expresiva. Estos resultados podrían indicar que se trata de un proceso de habituación debido al paso del tiempo. Puede ser interesante señalar que, en el caso de la ansiedad, los niveles iniciales son elevados tanto en el grupo experimental como en el control. Este hecho en ambos grupos podría sugerir que el alumnado que participó estuvo viviendo algún acontecimiento estresante, por lo que ese proceso de habituación puede deberse a circunstancias ajenas a las del proceso experimental.

Los pensamientos intrusivos y de evitación no se vieron afectados por la RE. Se esperaba que un procedimiento en el que se pretende recuperar traumas vividos para 
mejorar la salud tendría un reflejo en los efectos directos del propio trauma (Baikie et al., 2012). Sin embargo, tal y cómo se ha verificado en otras investigaciones (Travagin et al., 2015), esos efectos se producen en personas que informaron de una gravedad moderada de los síntomas de estrés postraumático. En nuestro caso, las puntuaciones previas en los pensamientos intrusivos y de evitación están alejadas de ser puntuaciones clínicamente significativas.

Finalmente, este trabajo pretendía ofrecer datos sobre el papel modulador de las variables personales de optimismo y alexitimia. De los resultados encontrados, el nivel de alexitimia, no pareció modular significativamente los resultados. Apelando a la significación clínica de los resultados (Martínez-Sánchez, 1996), el punto de corte para la puntuación de alexitimia se suele situar en una puntuación muy superior a los datos disponibles de nuestra muestra. Probar sus efectos moduladores con muestras que alcanzaran niveles de alexitimia clínicamente relevantes y posiblemente tomar en cuenta también el factor edad podría mejorar nuestros resultados (García-Sevilla, Méndez, Bermúdez, y Martínez, 2016; Méndez et al., 2015).

Este trabajo presenta ciertas limitaciones: se ha trabajado con una muestra de estudiantes, con participación voluntaria, sin conocer su historial de estrés postraumático y las implicaciones que pudiera tener el hecho recordado para su ajuste personal. Además, no se ha controlado la gravedad del trauma reexperimentado por cada participante. Se precisarían datos de seguimiento para confirmar que los beneficios de la utilización de la escritura expresiva se mantienen a lo largo del tiempo.

En suma, la promoción de la escritura de eventos traumáticos como técnica terapéutica parece prematura, por lo menos como técnica única, ya que dicha práctica no ha sido suficientemente justificada por los resultados empíricos (Koschwanez et al. 2013). Los resultados del presente estudio, junto con los de investigaciones anteriores, sugieren que, en el mejor de los casos, su uso debe limitarse a servir como un complemento a otros tratamientos apoyados empíricamente (Sloan, Sawyer, Lowmaster, Wernick, y Marx, 2015).

\section{REFERENCIAS}

Arancibia, M., y Behar, R. (2015). Alexitimia y depresión: evidencia, controversias e implicancias. Revista Chilena de Neuro-Psiquiatría, 53, 24-34.

Baguena, M., Villarroya, E., Beleña, A., Díaz, A., Roldan, C., y Reig, R. (2001). Propiedades psicométricas de la versión española de la Escala Revisada de Impacto del Estresor (EIE-R). Análisis y Modificación de Conducta, 27, 581-604.

Baikie, K.A. (2008). Who does expressive writing work for? Examination of alexithymia, splitting, and repressive coping style as moderators of the expressive writing paradigm. British Journal of Health Psychology, 13, 61-66. doi: 10.1348/135910707X250893 
Baikie, K.A., Geerligs, L., y Wilhelm, K. (2012). Expressive writing and positive writing for participants with mood disorders: An online-randomized controlled trial. Journal of Affective Disorders, 136(3), 310-319. doi: 10.1016/j.jad.2011.11.032

Blasio, P.D., Camisasca, E., Caravita, S.C.S., Ionio, C., Milani, L., y Valtolina, G.G. (2015). The effects of expressive writing on postpartum depression and posttraumatic stress symptoms. Psychological Reports, 117, 856-882. doi: 10.2466/02.13.PR0.117c29z3

Butcher, H.K., Gordon, J.K., Ko, J.W., Perkhounkova, Y., Cho, J.Y., Rinner, A., y Lutgendorf, S. (2016). Finding Meaning in Written Emotional Expression by Family Caregivers of Persons with Dementia. American Journal of Alzheimer's Disease and Other Dementias. doi: $10.1177 / 1533317516660611$

Clyman, J.A., y Pachankis, J.E. (2014). The relationship between objectively coded explanatory style and mental health in the stigma-related narratives of young gay men. Psychology of Men \& Masculinity, 15(1), 110. doi: 10.1037/a0031500

Frattaroli, J. (2006). Experimental Disclosure and Its Moderators: A Meta-Analysis. Psychological Bulletin, 132, 823-865. doi: 10.1037/0033-2909.132.6.823

García-Sevilla, J., Méndez, I., Bermúdez, A.M., y Martínez, J.P. (2016). Estudio comparativo de alexitimia en personas institucionalizadas versus aula de mayores. European Journal of Investigation in Health, Psychology and Education, 6, 5-14.

Gil, F., Oberst, U., Del Valle, G., y Chamarro, A. (2015). Nuevas tecnologías ¿Nuevas patologías? El smartphone y el fear of missing out. Aloma: Revista de Psicologia, Ciències de l'Educació i de l'Esport, 33, 77-83.

Horowitz, M.J., Wilner, N., y Alvarez, W. (1979). Impact of Event Scale: A measure of subjective stress. Psychosomatic Medicine, 41, 209-218. doi: 10.1097/00006842-197905000-00004

Ironson, G., O'Cleirigh, C., Leserman, J., Stuetzle, R., Fordiani, J., Fletcher, M., y Schneiderman, N. (2013). Gender-specific effects of an augmented written emotional disclosure intervention on posttraumatic, depressive, and HIV-disease-related outcomes: a randomized, controlled trial. Journal of Consulting and Clinical Psychology, 81, 284298. doi: $10.1037 / \mathrm{a} 0030814$

Kállay, É. (2015). Physical and Psychological Benefits of Written Emotional Expression. European Psychologist, 20, 242-251. doi: 10.1027/1016-9040/a000231

Koschwanez, H.E., Kerse, N., Darragh, M., Jarrett, P., Booth, R.J., y Broadbent, E. (2013). Expressive writing and wound healing in older adults: a randomized controlled trial. Psychosomatic Medicine, 75, 581-590. doi: 10.1097/PSY.0b013e31829b7b2e

Krpan, K.M., Kross, E., Berman, M.G., Deldin, P.J., Askren, M.K., y Jonides, J. (2013). An everyday activity as a treatment for depression: The benefits of expressive writing for people diagnosed with major depressive disorder. Journal of Affective Disorders, 150, 1148-1151. doi: 10.1016/j.jad.2013.05.065

Lafont, J., y Oberle, C.D. (2014). Expressive writing effects on body image: Symptomatic versus asymptomatic women. Psychology, 5, 431-440. doi: 10.4236/psych.2014.55053

Lumley, M.A., Keefe, F.J., Mosley-Williams, A., Rice, J.R., McKee, D., Waters, S.J., Partridge, R. T., Carty, J.N., Coltri, A.M., Kalaj, A., Neely, L.C., Pahssen, J.K., Connelly, M.A., Bouaziz, Y.B., Riordan, P.A., y Cohen, J.L. (2014). The effects of written emotional disclosure and coping skills training in rheumatoid arthritis: A randomized clinical trial. Journal of Consulting and Clinical Psychology, 82(4), 644. doi: 10.1037/a0036958

Mackenzie, C.S., Wiprzycka, U.J., Hasher, L., y Goldstein, D. (2008). Seeing the glass half full: Optimistic expressive writing improves mental health among chronically stressed caregivers. British Journal of Health Psychology, 13, 73-76. doi: $10.1348 / 135910707 X 251153$ 
Meads, C., y Nouwen, A. (2005). Does emotional disclosure have any effects? A systematic review of the literature with meta-analyses. International Journal of Technology Assessment in Health Care, 21, 153-164. doi: 10.1017.S026646230505021X

Méndez, I., García-Sevilla, J., Martínez, J.P., Bermúdez, A.M., Pérez, P., y García-Munuera, I. (2015). Alexitimia, resiliencia y creencias paranormales en personas mayores en un centro institucionalizado. European Journal of Investigation in Health, Psychology and Education, 5, 209-219.

Merz, E.L., Fox, R.S., y Malcarne, V.L. (2014). Expressive writing interventions in cancer patients: a systematic review. Health Psychology Review, 8, 339-361. doi: 10.1080/17437199.2014.882007

Meston, C.M., Lorenz, T.A., y Stephenson, K.R. (2013). Effects of expressive writing on sexual dysfunction, depression, and PTSD in women with a history of childhood sexual abuse: Results from a randomized clinical trial. The Journal of Sexual Medicine, 10, 21772189. doi: $10.1111 /$ jsm. 12247

Niles, A.N., Haltom, K.E., Mulvenna, C.M., Lieberman, M.D., y Stanton, A.L. (2014). Effects of Expressive Writing on Psychological and Physical Health: The Moderating Role of Emotional Expressivity. Anxiety, Stress, and Coping, 27, 1-17. doi: 10.1080/10615806.2013.802308.

Nolla, M.C., Queral, R., y Miró, J. (2014). Las escalas PANAS de afecto positivo y negativo: Nuevos datos de su uso en personas mayores. Revista de Psicopatología y Psicología Clínica, 19, 15-21.

Núñez, J.C., Amieiro, N., Álvarez, D., García, T., y Dobarro, A. (2015). Self-Regulated Learning from Texts (ARATEX-R) Assessment Scale. European Journal of Education and Psychology, 8, 9-22.

O'Connor, D.B., Hurling, R., Hendrickx, H., Osborne, G., Hall, J., Walklet, E., Whaley, A., y Wood, H. (2011). Effects of written emotional disclosure on implicit self- esteem and body image. British Journal of Health Psychology, 16, 488-501. doi: 10.1348/135910710X523210

Peasley-Miklus, C.E., Panayiotou, G., y Vrana, S.R. (2016). Alexithymia predicts arousal-based processing deficits and discordance between emotion response systems during emotional imagery. Emotion, 16(2), 164. doi: 10.1037/emo0000086

Peñate, W., Del Pino-Sedeño, T., y Bethencourt, J.M. (2010). El efecto diferencial de escribir sobre un mismo trauma o sobre diferentes traumas en el bienestar psicológico y el estado de salud autoinformado. Universitas Psychologica, 9, 433-445.

Scheier, M.F., Carver, C.S., y Bridges, M.W. (1994). Distinguising optimism from neuroticism (and trait anxiety, self mastery and self esteem): A reevaluation of the Life Orientation Test. Journal of Personality and Social Psychology, 67, 1063-1078. doi: 10.1037/0022-3514.67.6.1063

Sloan, D.M., Sawyer, A.T., Lowmaster, S.E., Wernick, J., y Marx, B.P. (2015). Efficacy of Narrative Writing as an Intervention for PTSD: Does the Evidence Support Its Use? Journal of Contemporary Psychotherapy, 45(4), 215-225. doi: 10.1007/s10879-014-9292-x

Smyth, J.M. (1998). Written emotional expression: effect sizes, outcome types, and moderating variables. Journal of Consulting and Clinical Psychology, 66, 174. doi: 10.1037/0022006X.66.1.174

Sternberg, R.J. (2015). Successful intelligence: A model for testing intelligence beyond IQ tests. European Journal of Education and Psychology, 8, 76-84. doi: 10.1016/j.ejeps.2015.09.004 
Taber, J.M., Klein, W.M., Ferrer, R.A., Kent, E.E., y Harris, P.R. (2016). Optimism and spontaneous self-affirmation are associated with lower likelihood of cognitive impairment and greater positive affect among cancer survivors. Annals of Behavioral Medicine, 50, 198-209. doi: 10.1007/s12160-015-9745-9

Taylor, G.J., Bagby, R.M., y Parker, J.D.A. (1997). Disorders of affect regulation: Alexithymia in medical and psychiatric illness. Cambridge: Cambridge University Press. doi: 10.1017/CBO9780511526831

Travagin, G., Margola, D., y Revenson, T.A. (2015). How effective are expressive writing interventions for adolescents? A meta-analytic review. Clinical Psychology Review, 36, 42-55. doi: 10.1016/j.cpr.2015.01.003

Unterhitzenberger, J., y Rosner, R. (2014). Lessons from writing sessions: A school-based randomized trial with adolescent orphans in Rwanda. European Journal of Psychotraumatology, 5. doi: 10.3402/ejpt.v5.24917

Vera-Villarroel, P., y Celis-Atenas, K. (2014). Positive and Negative Affect as Mediator of the Relatioship Optimism-Health: Evaluation of a Structural Model. Universitas Psychologica, 13, 1017-1026. doi: 10.11144/Javeriana.UPSY13-3.apnm

Watson, D., Clark, L.A., y Tellegen, A. (1988). Development and validation of brief measures of positive and negative affect: The PANAS scales. Journal of Personality and Social Psychology, 54, 1063-1070. doi: 10.1037/0022-3514.54.6.1063

Zigmond, A.S., y Snaith, R.P. (1983). The Hospital Anxiety and Depression Scale. Acta Pschyatrica Scandinavica, 67, 361-370. doi: 10.1111/j.1600-0447.1983.tb09716.x

Recibido: 10 de marzo de 2016

Recepción Modificaciones: 27 de julio de 2016 Aceptado: 29 de julio de 2016 